\title{
Métodos exatos para resolver o problema de programação da tripulação
}

\author{
Gustavo Peixoto Silva'; Marcone Jamilson Freitas Souza²; Jorge von Atzingen³
}

\begin{abstract}
Resumo: Este trabalho tem como objetivo implementar um método de otimização para o Problema da Programação de Tripulações (PPT), o qual visa determinar um conjunto de jornadas de trabalho para as tripulações, de tal forma que a programação dos veículos seja realizada com o menor custo possível. Como restrições, cada jornada deve atender à legislação e à convenção coletiva de trabalho do setor. Neste trabalho são apresentadas e comparadas quatro diferentes metodologias de geração de colunas para o PPT, definindo assim problemas de programação linear inteira com variáveis binárias. A primeira metodologia consiste em definir um intervalo de tempo durante o qual poderá ocorrer a troca de tripulações. Na segunda metodologia as jornadas possuem pelo menos um dado tempo mínimo de duração. Na terceira metodologia é implementada a heurística de Chvátal. A quarta metodologia apresenta a implementação de um método híbrido de geração de colunas para o PPT. Neste trabalho apresentam-se resultados comparativos obtidos com a aplicação das metodologias a problemas reais.
\end{abstract}

\begin{abstract}
The purpose of this work is the implementation of an optimization method for the bus crew scheduling problem (BCS), which aims to determine a set of workdays to the crews, so that the vehicle scheduling could be performed with the minimum operational cost. The constraints of this problem should comply with the labor agreement and operational rules. In this paper it is presented and compared four different methodologies of columns generation for the BCS, defining as these problems of linear programming integer with binary variables. The first methodology consists of determining a time interval during which the crew's exchange can happen. In the second methodology the journey owns at least a minimum time length. In the third methodology the Chvátal's heuristics is implemented. The fourth methodology presents the implementation of a hybrid method of columns generation for the BCS. In this paper, comparative results on real-world problems are presented.
\end{abstract}

\section{INTRODUÇÃO}

O Problema de Geração da Escala de Motoristas e Cobradores Urbanos é denominado no meio científico de Problema da Programação de Tripulações (PPT) de um Sistema de Transporte Público. Este problema consiste em determinar o número mínimo necessário de tripulações (motorista e cobrador), tal que a programação dos veículos (conjunto de viagens atribuídas a cada veículo) seja realizada com sucesso. A solução deste problema também envolve o seqüenciamento das atividades de cada tripulação, gerando um conjunto de jornadas de trabalho, cujo custo operacional total seja mínimo. Este problema se torna complexo devido às restrições operacionais vigentes nas empresas e às cláusulas contidas nas Convenções Coletivas de Trabalho.

A importância do PPT se deve ao fato de que uma boa parcela dos custos das empresas é composta pelo custo da mão-de-obra em operação. Uma vez que o sistema, em tese, deve ser mantido pelos seus usuários, uma redução no custo de mão-de-obra pode significar um benefício para milhares de pessoas. A defi-

\footnotetext{
${ }^{1}$ Gustavo Peixoto Silva, Universidade Federal de Ouro Preto. Ouro Preto, MG, Brasil (e-mail: gustavo@iceb.ufop.br).

${ }^{2}$ Marcone Jamilson Freitas Souza, Universidade Federal de Ouro Preto. Ouro Preto, MG, Brasil (e-mail: marcone@iceb.ufop.br)

${ }^{3}$ Jorge von Atzingen, Universidade Federal de Ouro Preto. Ouro Preto, MG, Brasil (e-mail: jorge.reis@poli.usp.br).
}

Este artigo é parte de TRANSPORTES, volume XIV, número 1 , junho de 2006. ISSN: 1415-7713. nição de uma escala de tripulações econômica é um problema de otimização e várias técnicas têm sido desenvolvidas para resolver o PPT. Pela sua importância e grau de dificuldade, este problema desperta grande interesse no meio científico. Neste trabalho são apresentadas e comparadas quatro metodologias para a resolução do PPT.

Esse tema tem sido largamente estudado e seus resultados são geralmente utilizados nos países mais desenvolvidos. A abordagem mais explorada é aquela que formula o PPT como um problema de recobrimento ou de particionamento (set covering ou set partitioning model) e utiliza a técnica de geração de colunas para resolvê-lo (Smith e Wren 1988, Desrochers e Soumis 1989, Desrochers et al. 1992, Fores et al. 1999, Barnhart et al. 1998, Friberg e Haase 1999, Fores 1996). A variedade de trabalhos deriva das diferentes maneiras de gerar as colunas e diferentes metodologias para resolver o problema.

Desrochers e Soumis (1989) propõem uma abordagem de geração de colunas para o PPT que decompõe o problema em duas partes: um problema principal que é o problema de recobrimento e um subproblema de caminho mínimo. O problema de recobrimento escolhe uma programação dentre as jornadas factíveis previamente conhecidas. O subproblema é usado para encontrar uma nova jornada factível que melhore a solução corrente do problema de recobrimento. No modelo de recobrimento são consideradas as restrições globais do problema, tais como os números máximo e mínimo de jornadas contínuas e o número máximo de 
tripulações. Esse problema é resolvido pelo método Simplex e fornece uma relaxação linear do problema de recobrimento. Uma vez resolvido o problema principal, as novas colunas são geradas pelo algoritmo de caminho mínimo com restrições adicionais. Nesse subproblema, cada caminho factível existente na rede que liga a origem até o destino representa uma jornada viável. A função objetivo é definida de tal forma que o caminho tenha o menor custo marginal possível.

O trabalho de Freling et al. (2001) formula o PPT como um problema de recobrimento e o resolve combinando a técnica de relaxação lagrangeana com a de geração de colunas. Haase et al. (2001) usa uma aproximação exata para resolver o problema de programação da tripulação integrado com o problema de programação de veículos. O problema é resolvido usando o método branch-and-bound e o método de planos de cortes juntamente com a geração de colunas. Pedrosa e Constantino (2001) apresentam um modelo de recobrimento em que a mão-de-obra é dividida em grupos usando uma lista circular e o PPT é resolvido usando uma heurística construtiva com geração de colunas. Valouxis e Housos (2002) dividem a tripulação em níveis, de forma a reduzir as dimensões do PPT. Estes níveis são baseados em um algoritmo de emparelhamento das trocas de tripulação. Yunes et al. (1999) utiliza programação inteira e geração de colunas para resolver o PPT formulado como um problema de particionamento.

O trabalho de Desrochers e Soumis (1989) constitui a base do método HASTUS (Rousseau e Blais, 1985), que consiste no sistema comercial para a programação de tripulações, desenvolvido no centro de pesquisa GERARD da Universidade de Montreal.

Um outro sistema comercial de referência internacional é o TRACS II (Wren et al. 1994) desenvolvido pelo Grupo de Programação de Restrições e Pesquisa Operacional da Universidade de Leeds no Reino Unido. O sistema TRACS II é uma versão atual do IMPACS e vem sendo aprimorado e adaptado para resolver também o problema de programação de tripulações de trens (Wren et al. 1994, Fores et al. 1999). Fores et al. (2002) apresenta uma heurística de programação linear inteira que incorporada ao TRACS II possibilitou a resolução de problemas com maior complexidade. O sistema IMPACS tem como base o trabalho de Smith e Wren (1988) que emprega o modelo de recobrimento associado a um método de busca branch-and-bound para a obtenção de uma solução inteira para o PPT. Nesse caso, para diminuir o número de colunas, são consideradas as características do problema, gerando apenas as colunas que se referem a jornadas de trabalho factíveis e similares àquelas praticadas pelas empresas operadoras do sistema. Dessa forma, o espaço de busca do Simplex é reduzido con- sideravelmente, permitindo a resolução do problema em um tempo razoável de processamento computacional. Este trabalho serviu como motivação para o presente artigo.

A primeira metodologia utiliza o conceito de janela de troca, que consiste em definir um intervalo de tempo no qual poderá ocorrer a troca de tripulações. Com esta metodologia são geradas apenas as jornadas cujo tempo de trabalho se aproximam do tempo máximo de trabalho definido pela legislação trabalhista. Na segunda metodologia as jornadas possuem pelo menos um dado tempo mínimo de duração. Este tempo mínimo é atingido agrupando as tarefas que estão no início da operação de cada veículo, assim como as tarefas do final de cada veículo. Desta forma foi obtida uma grande redução no número de jornadas viáveis. Na terceira metodologia é implementada a heurística de Chvátal usando o conceito de custo reduzido, que seleciona as colunas que possuem o menor custo operacional. Deste modo, não foi necessário submeter o problema a um pacote de otimização. A quarta metodologia apresenta a implementação de um método híbrido de geração de colunas para o PPT. Para reduzir o número de colunas do problema, é realizada uma adaptação da heurística de Chvátal para o problema de particionamento.

As implementações foram testadas com dados reais de uma empresa que opera em Belo Horizonte, contribuindo com a área de pesquisa no sentido de colocar em prática técnicas de otimização, acumular conhecimentos e desenvolver ferramentas que permitem reduzir os custos da mão-de-obra operacional e conseqüentemente do sistema de transporte público como um todo.

Na próxima seção é apresentado o modelo de particionamento para o PPT. Na seção 3 é realizada a descrição das metodologias propostas para reduzir a dimensão do modelo que representa o PPT como um modelo de particionamento. Os resultados de um estudo de caso são apresentados na seção 4. Finalmente são apresentadas as conclusões e uma proposta de continuidade do trabalho.

\section{O MODELO DE PARTICIONAMENTO PARA O PPT}

A programação da tripulação tem como dados de entrada a programação dos veículos, previamente definida. A programação dos veículos define os blocos dos veículos, ou seja, o conjunto de viagens a ser executado por cada veículo da frota. Os blocos são divididos em tarefas, que são seqüências de viagens que devem ser executadas por uma única tripulação, devido aos motivos operacionais esclarecidos a seguir.

No modelo de particionamento, apresentado em (1) 
- (4), cada linha da matriz está relacionada a uma única tarefa, enquanto as colunas se relacionam com as possíveis jornadas de trabalho. Assim, o elemento $a_{i j}$ $=1$ se a tarefa $i$ faz parte da jornada $j$ e $a_{i j}=0$ caso contrário, para $i=1, \ldots, m, j=1, \ldots, n$. A variável $x_{j}=1$ se a jornada $j$ faz parte da solução, ou seja, da escala diária da empresa e $x_{j}=0$ caso contrário (restrição 4 ). $\mathrm{O}$ vetor $b$ tem todos os elementos iguais a 1 , garantindo através da restrição (2) que cada tarefa seja executada uma única vez. Ao final das $m$ tarefas é acrescida a linha $m+1$ que se refere às jornadas do tipo dupla pegada, ou seja, aquelas jornadas compostas por dois períodos de trabalho, geralmente nos horários de pico, com um intervalo entre eles maior do que duas horas. Se a jornada $j$ for desse tipo, então $a_{m+1, j}=1$, e $a_{m+1 j}=$ 0 caso contrário. Nessa linha a componente $b_{m+1}$ do vetor $b$ tem o número máximo permitido de jornadas do tipo dupla pegada, que em geral fica em torno de $10 \%$ do número total de tripulantes da solução da empresa. A restrição (3) garante que esse número nunca seja ultrapassado.

$$
\begin{gathered}
\operatorname{Min} \sum_{j=1}^{n} c_{j} \cdot x_{j} \\
\sum_{j=1}^{n} a_{i j} \cdot x_{j}=1 \quad \forall i=1, \ldots, m \\
\sum_{j=1}^{n} a_{m+1, j} \cdot x_{j} \leq \max _{-} \text {dupla_pegada } \\
x_{j} \in\{0,1\} \quad \forall j=1, \ldots, m
\end{gathered}
$$

O custo de cada jornada é calculado em função do tempo total que a tripulação é remunerada e não está dirigindo o veículo, dito tempo ocioso e do total de horas extras contidas na jornada.

\section{AS METODOLOGIAS PROPOSTAS}

As metodologias adotadas se concentram na geração das jornadas de trabalho que compõem o modelo de particionamento. Nesse sentido, tira-se proveito das características particulares do PPT para reduzir o número de jornadas a serem consideradas no modelo. Por outro lado, tal redução deve contemplar os diversos tipos de jornadas para não comprometer a qualidade da solução obtida. As jornadas de trabalho são combinações de tarefas dos veículos de uma mesma linha. Entretanto, foi considerada a possibilidade de uma tripulação executar tarefas de veículos distintos, ou seja, que uma tripulação realize a troca de veículos que operam em uma mesma linha.

Inicialmente é realizada a geração das tarefas. Esta fase tem como entrada os dados referentes às viagens diárias de cada veículo das respectivas linhas de responsabilidade da empresa. As viagens de cada veículo são agrupadas em tarefas, que são seqüências de viagens que devem ser executadas por uma única tripulação, ao final dessa etapa todas as viagens da empresa são transformadas em tarefas. Isso se deve ao fato de não haver entre as viagens condições necessárias para a realização da troca da tripulação, ou seja, o seu rendimento por uma outra tripulação. As condições necessárias para a troca de duas tripulações definem uma oportunidade de troca (OT), que consiste em um intervalo de tempo mínimo entre duas viagens (tempo de troca), o qual deve ocorrer em um local apropriado para a execução da troca (ponto de troca). As viagens que constituem uma tarefa possuem as seguintes características:

- Tempo de terminal entre as viagens menor que cinco minutos (parâmetro de entrada);

- Final de uma tarefa ocorre apenas nos pontos onde existe um fiscal da empresa;

- A primeira viagem da tarefa tem início em um ponto onde existe um fiscal, ou na garagem da empresa;

- A última viagem da tarefa tem término em um ponto onde existe um fiscal, ou na garagem da empresa.

As restrições trabalhistas contempladas neste trabalho são:

- Folga corrida de no mínimo 15 minutos e folga total de no mínimo 30 minutos;

- Tempo máximo de trabalho igual a 9 horas e 10 minutos;

- Tempo mínimo entre o final da jornada e seu início no dia seguinte de 11 horas;

- Jornada normal de trabalho com duração de 7 horas e 10 minutos.

\subsection{Procedimento de geração de janelas de troca}

A primeira etapa é a geração das janelas de troca, as quais são geradas por um processo similar àquele descrito por Smith e Wren (1988), no qual são combinadas as tarefas a fim de se obterem intervalos de tempo nos quais deverá ocorrer a troca da tripulação dos veículos de uma dada linha. Esse intervalo é definido tendo em vista as seguintes características:

- Tempo máximo de trabalho da solução da empresa;

- Tempo médio de trabalho da solução da empresa.

Para melhor entendimento do processo, considere o seguinte exemplo da seleção de um conjunto de janela de troca: suponha que um ônibus deixe a garagem às 07:00 horas e retorne às 21:00 horas, com oportunidades de troca a cada meia hora. Suponha também que o 
tempo máximo de trabalho seja de 04:45 horas e o tempo médio de trabalho de 03:45 horas. A partir de 07:00 horas acrescenta-se 04:45 horas resultando no horário 11:45. Entretanto, visto que não existe oportunidade de troca às 11:45 horas, é considerada a última oportunidade anterior, ou seja, às 11:30 horas como o término de uma janela. A partir do primeiro ponto, definido às 11:30 horas, é acrescido o tempo médio de trabalho, ou seja, 03:45 horas, resultando às 15:15 horas. Como essa não é uma OT, recua-se até a primeira OT imediatamente anterior, definindo o término da segunda janela de troca. $\mathrm{O}$ processo se repete até o final da operação (Figura 1).

Trabalhando no sentido reverso parte-se das 21:00 horas e, de modo semelhante ao direto, são produzidos os seguintes horários de término das janelas: 16:30, 13:00, e 09:30 (Figura 2). Combinando os dois grupos de tempos marcados, resulta nas janelas de troca 09:30-11:30, 13:00-15:00, e 16:30-18:30 (Figura 3).

Uma vez definidas as janelas de trocas dos diversos veículos de uma dada linha, essas são combinadas para se obterem as janelas de troca da linha. O procedimento tem as seguintes características:

- O início da primeira janela corresponde ao início da janela com horário mais cedo;

- O término da última janela corresponde ao término da janela com horário mais tarde;

- Adiciona-se o tempo médio de trabalho da linha ao horário de início, marcando o meio da janela seguinte;

- Subtrai-se o tempo médio de trabalho da linha ao horário de término, marcando o meio da última janela.

As extremidades das janelas são calculadas somando-se e subtraindo-se um parâmetro de entrada, denominado fator de alargamento da janela de troca. $\mathrm{O}$ resultado desta etapa é um conjunto de janelas de troca para cada linha, como o exemplo da Figura 3. A partir das janelas de trocas das respectivas linhas são geradas jornadas, cujas trocas de tripulação se dão sempre no interior dos intervalos de troca.

A segunda etapa é a geração das tarefas fixas e rotativas. As janelas de troca são intervalos nos quais devem ocorrer as trocas das tripulações. Assim, as tarefas de um dado veículo são combinadas seqüencialmente até atingirem um tempo mínimo de trabalho, cujo horário final esteja dentro da janela de troca. A partir desse ponto existe a possibilidade de combinar as tarefas inteiramente contidas na janela de troca ou de encerrar a jornada de trabalho da tripulação desse veículo.

A seqüência de tarefas que parte do início da operação ou do interior de um intervalo de troca e vai até atingir o interior da próxima janela de troca ou o final da operação, é agrupada em uma única tarefa denominada tarefa fixa. As tarefas inteiramente contidas no interior de uma janela de troca são denominadas tarefas rotativas.

Como resultado, esta etapa define as tarefas fixas e rotativas de cada veículo da frota, que combinadas dão origem às diferentes jornadas de trabalho.

Na última etapa são geradas todas as possíveis jornadas de trabalho em relação aos veículos da empresa. Uma jornada de trabalho será composta por uma tarefa fixa sozinha ou por uma fixa com uma ou mais tarefas rotativas. Todas as possíveis combinações são geradas, entretanto apenas as jornadas que satisfazem as restrições legais são incluídas no modelo. A jornada composta por duas tarefas fixas é denominada uma jornada do tipo dupla pegada.

O custo operacional $c_{j}$ de cada jornada é calculado de acordo com a seguinte expressão:

$$
C_{j}=T_{h_{-} \text {extra }} \cdot P_{h_{-} \text {extra }}+T_{\text {ocioso }} \cdot P_{\text {ocioso }}
$$

sendo,

$c_{j}$ : custo da coluna $j$,

$T_{h_{\_} \text {extra }}:$ número de horas extras da coluna $j$,

$P_{h_{-} \text {extra }}$ : peso dado a cada minuto de hora extra presente na coluna $j$,

$T_{\text {ocioso }}$ : número de horas ociosas da coluna $j \mathrm{e}$

$P_{\text {ocioso }}$ : peso dado a cada minuto de hora ociosa da coluna $j$.

Cada jornada define uma coluna da matriz de restrições e um coeficiente na função de custo. Na Tabela 1 é apresentado um exemplo de uma matriz gerada segundo essa metodologia, na qual as linhas correspondem às tarefas de cada veículo. A Tarefa 0101 corresponde à primeira tarefa do modelo, a qual pertence ao veículo 1, enquanto a Tarefa 0302 é a terceira tarefa do modelo que pertence ao veículo 2 . A coluna 1 corresponde à jornada composta apenas pela Tarefa 0101, e a coluna 6 representa a jornada que realiza as Tare-

07:00>------------------11:30>------------------15:00>------------------18:30>------------------<21:00

Figura 1: Tempos que determinam o término das janelas (sentido direto)

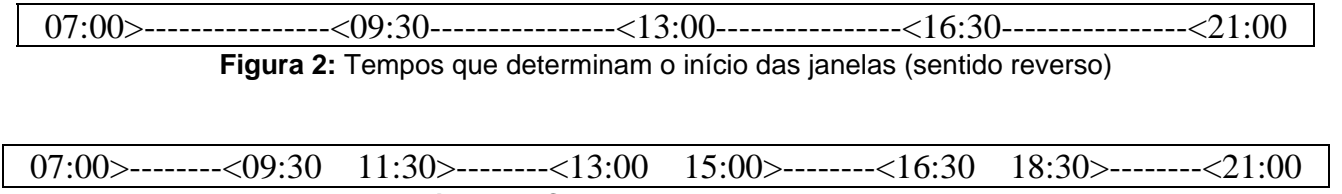

Figura 3: Conjunto de Janelas de Troca 
Tabela 1: Exemplo de uma Matriz gerada pelo programa Geratarefa

\begin{tabular}{lrrrrrrrrrrrr}
\hline Jornadas & $\boldsymbol{J 1}$ & $\boldsymbol{J} 2$ & $\boldsymbol{J 3}$ & $\boldsymbol{J 4}$ & $\boldsymbol{J 5}$ & $\boldsymbol{J 6}$ & $\boldsymbol{J 7}$ & $\boldsymbol{J 8}$ & $\boldsymbol{J 9}$ & $\boldsymbol{J 1 0}$ & $\boldsymbol{J 1 1}$ & $\boldsymbol{J 1 2}$ \\
\hline Tarefa 0101 & 1 & 0 & 0 & 1 & 0 & 1 & 0 & 0 & 1 & 0 & 1 & 1 \\
Tarefa 0201 & 0 & 0 & 0 & 1 & 0 & 1 & 0 & 1 & 0 & 1 & 0 & 0 \\
Tarefa 0302 & 0 & 0 & 0 & 0 & 0 & 1 & 0 & 1 & 0 & 1 & 0 & 0 \\
Tarefa 0401 & 0 & 1 & 0 & 0 & 0 & 0 & 0 & 1 & 0 & 0 & 0 & 1 \\
Tarefa 0502 & 0 & 0 & 1 & 0 & 1 & 0 & 0 & 0 & 0 & 1 & 0 & 0 \\
Tarefa 0602 & 0 & 0 & 0 & 0 & 1 & 0 & 1 & 0 & 1 & 0 & 0 & 0 \\
Tarefa 0701 & 0 & 0 & 0 & 0 & 0 & 0 & 1 & 0 & 0 & 0 & 1 & 0 \\
\hline
\end{tabular}

fas 0101, 0201 e 0302. No primeiro exemplo a tripulação opera um único veículo, enquanto no segundo a tripulação atuará em dois veículos distintos. Essa estratégia permite uma flexibilização na operação e conseqüentemente uma redução nos seus custos, sem no entanto propor uma alteração drástica na filosofia de trabalho da empresa.

Os tipos de jornadas possíveis nesta metodologia são:

- Fixa sozinha: ocorre quando a tripulação executa somente as tarefas contidas em uma única fixa. Este tipo de jornada ocorre nas jornadas $\mathrm{J} 1$, J2 e J3;

- Fixa com Rotativa: é composta de uma ou mais rotativas e de uma fixa do mesmo veículo. Este tipo de jornada ocorre nas jornadas J4, J5, J6, J7 e J8;

- Fixa com Rotativa de outro veículo: é caracterizada pela combinação de uma fixa de um veículo com uma ou mais rotativas de outro veículo. A fixa e a rotativa não precisam ser necessariamente da mesma linha. Neste tipo de jornada ocorre o que se denomina de troca de veículo, podendo também ocorrer à troca de linha. Como exemplo podemos citar as jornadas J9 e J10;

- Duas fixas de veículos diferentes: ocorre quando as tarefas fixas estão separadas por um intervalo maior ou igual a duas horas. Essa é uma dupla pegada, na qual pode ou não ocorrer troca de linha. Este tipo de jornada pode ser observado em J11;

- Duas Fixas do mesmo veículo: é a combinação de duas fixas do mesmo veículo com um intervalo intermediário de pelo menos duas horas. Este tipo de jornada ocorre na coluna J12.

\subsection{Procedimento de Agrupamento de Tarefas}

Para reduzir o número de jornadas e garantir uma du- ração mínima de cada jornada, as tarefas que se localizam no início e no final de cada veículo foram agrupadas em uma única tarefa da seguinte forma:

- A primeira tarefa de cada veículo é combinada com as tarefas seguintes deste veículo até atingir o tempo mínimo de trabalho;

- Da mesma forma, a última tarefa de cada veículo é combinada com as tarefas anteriores até atingir o tempo mínimo de trabalho.

Dessa forma foi agrupada parte das tarefas, diminuindo o número de combinações possíveis de serem realizadas, pois quanto maior o número de tarefas maior será o número de combinações. A Tabela 2 contém o número de tarefas de cada linha antes e depois da união das tarefas iniciais e finais de cada veículo.

Pelos dados da Tabela 2 pode-se observar que houve uma grande redução no número de tarefas de cada linha atingindo uma redução de 55\% no número total de tarefas.

Em seguida foram consideradas todas as possíveis combinações de tarefas gerando as jornadas de trabalho. Cada jornada é avaliada e apenas as jornadas viáveis, ou seja, aquelas que satisfazem as restrições trabalhistas, são incluídas no modelo. Foram gerados os seguintes tipos de jornadas de trabalho:

- Dupla Pegada: ocorre quando a tripulação executa parte das tarefas de uma determinada jornada e após uma folga corrida de pelo menos 2 horas, retorna para continuar a cumprir o restante das tarefas pertencentes à mesma jornada. Durante o período que a tripulação permanece parada, ela não tem remuneração;

- Pegada Simples: é quando uma tripulação executa todas as tarefas de uma determinada jornada com uma folga corrida menor que 2 horas. Neste caso deve haver uma folga para alimentação/repouso de 30 minutos incluída na jornada.

Tabela 2: Redução do número de tarefas devido ao processo de tempo mínimo

\begin{tabular}{lcccccccccccc}
\hline Número da Linha & 01 & 02 & 03 & 04 & 05 & 06 & 07 & 08 & 09 & 10 & 11 & Total \\
\hline Número de tarefas & 39 & 49 & 23 & 54 & 190 & 40 & 128 & 150 & 200 & 154 & 163 & 1190 \\
\hline Número reduzido de tarefas & 16 & 19 & 11 & 22 & 82 & 21 & 63 & 74 & 87 & 67 & 73 & 535 \\
\hline
\end{tabular}




\subsection{Heurística de Chvátal}

A heurística de Chvátal (1979) é um procedimento que constrói interativamente uma solução para o PPT. A cada iteração do método o conjunto de colunas é avaliado por uma função que leva em consideração o custo de cada coluna e o número de tarefas por ela coberta. Mais precisamente, cada coluna $j$ é avaliada pela expressão:

$$
\text { custo_relativo }{ }_{j}=\frac{c_{j}}{\text { total_tarefas_cobertas }}
$$

sendo: $c_{j}$ é o custo da coluna $j$; total_tarefas_cobertas representa o número de tarefas cobertas pela inserção da coluna $j$ na solução parcial e custo_relativo ${ }_{j}$ representa o custo relativo da coluna $j$.

Após a avaliação das colunas, aquela de menor custo relativo é inserida na solução parcial e excluída do conjunto de colunas candidatas. A seguir, todas as colunas candidatas remanescentes são novamente avaliadas, excluindo-se em cada coluna as tarefas já cobertas pelas colunas anteriormente inseridas no conjunto solução. Este procedimento se encerra quando todas as tarefas forem cobertas.

\subsection{Heurística de Seleção de Colunas}

Após a geração de todas as colunas, usando a metodologia do agrupamento de tarefas, as quais correspondem às jornadas de trabalho viáveis, é feita uma seleção de parte desse conjunto de forma a reduzir as dimensões da matriz e garantir que o modelo encontre uma solução ótima dentro de um tempo de processamento aceitável. A seleção das colunas é realizada tendo como base a heurística proposta por Chvátal (1979).

A cada coluna é atribuído um custo relativo calculado pela expressão (6), em seguida as colunas são ordenadas segundo seus custos relativos e aquelas que apresentam os menores valores irão compor as colunas da matriz do modelo de programação linear. Para garantir a existência de uma solução para o PPT, são adicionadas as jornadas de trabalho que possuem uma única tarefa. O número de jornadas de trabalho a ser considerado é definido como um parâmetro de entrada do sistema.

Este modelo foi testado com os dados reais de uma empresa de transporte público e os resultados são apresentados a seguir.

\section{RESULTADOS OBTIDOS}

Para validar as metodologias propostas, foram utilizados os dados reais de uma empresa que opera na cidade de Belo Horizonte. O cálculo da função de custo dada pela expressão (5), foi realizado utilizando-se os seguintes pesos: ociosidade $=40$ e hora extra $=50$. O tempo máximo de trabalho considerado foi de 09:10 (nove horas e dez minutos) e o tempo médio de trabalho foi de 07:33 (sete horas e trinta e três minutos). Os problemas associados aos testes foram resolvidos utilizando o pacote de otimização LINGO versão 7.0, em um microcomputador Athlon XP 2000 e 778 MB de RAM.

Nestes testes foi considerado apenas um grupo de linhas sob responsabilidade da empresa. Para cada metodologia (exceto a heurística de Chvátal) foi gerada uma matriz conforme o exemplo da tabela 1. Este problema foi submetido ao pacote de otimização e os resultados obtidos estão expostos nas tabelas 3, 4, 5 e 6.

Os resultados apresentados na Tabela 3 demonstram que as metodologias agrupamento de tarefas e seleção de colunas conseguiram obter os melhores resultados com baixos valores para o número de horas extras e para a quantidade de horas ociosas. A metodologia da janela de troca obteve uma solução um pouco pior apresentando 16 minutos a mais de hora extra e 106 minutos adicionais de horas ociosas. No entanto esta metodologia conseguiu obter estes resultados em apenas 2 segundos e também obteve um número reduzido de trocas de veículo. Este é um fato importante pois a empresa possui a cultura organizacional de evitar trocas de veículos. A metodologia Chvátal obteve o menor valor para a quantidade de

Tabela 3: Resultados obtidos usando tempo mínimo de trabalho igual a 5h00

\begin{tabular}{lccrr}
\hline & Janela de Troca & Agrupamento de Tarefas & Chvátal & Seleção de Colunas \\
\hline Número de colunas & 210 & 106.849 & 106.849 & 10.684 \\
Número de linhas & 44 & 46 & 46 & 46 \\
Função Objetivo & 70.580 & 65.540 & 77.400 & 65.540 \\
Tempo LINGO (segundos) & 1 & 357 & -117 & 30 \\
Tempo total (segundos) & 2 & 469 & 17 & 270 \\
Número de tripulações & 16 & 16 & 2 & 16 \\
Número de duplas pegadas & 3 & 2 & 9 & 2 \\
Número de trocas de veículo & 3 & 9 & 7 & 11 \\
Número de trocas de linhha & 0 & $00: 54$ & $00: 08$ & 10 \\
Horas extras & $01: 10$ & $26: 11$ & $32: 05$ & $00: 54$ \\
Horas ociosas & $27: 57$ & & & $26: 11$ \\
\hline
\end{tabular}


horas extras mas, por outro lado ela utiliza 17 tripulações e apresenta um número maior de horas ociosas do que as demais metodologias.

Os resultados apresentados na Tabela 4 mostram a eficácia da janela de troca que conseguiu minimizar o número de colunas geradas. Este fato é importante pois, devido ao aumento do número de colunas geradas, o agrupamento de tarefas não conseguiu resolver o problema. Conforme o tempo mínimo de trabalho for reduzido, maior será o número de colunas geradas e conseqüentemente aumentará a possibilidade de encontrar melhores soluções. Entretanto, conforme for maior o número de colunas, maior será a possibilidade de o computador utilizado nos teste não conseguir re- solver o problema.

Os resultados obtidos na Tabela 5 mostram que, devido ao maior número de colunas geradas, foi possível encontrar uma solução melhor que as soluções obtidas nas tabelas 3 e 4 . A seleção de colunas encontrou valores menores para a hora extra e para a hora ociosa. Isto ocorreu devido ao maior número de trocas de veículo e de trocas de linha apresentado na tabela acima.

Os resultados apresentados na Tabela 6 são a melhor solução obtida neste trabalho. A seleção de colunas conseguiu reduzir o número de tripulações necessárias para apenas 15 . No entanto, esta solução demorou aproximadamente duas horas e meia para ser obtida (um tempo computacional elevado quando compa-

Tabela 4: Resultados obtidos usando tempo mínimo de trabalho igual a 4h30

\begin{tabular}{|c|c|c|c|c|}
\hline & Janela de Troca & Agrupamento de Tarefas & Chvátal & Seleção de Colunas \\
\hline Número de colunas & 210 & 239.264 & 239.264 & 23.926 \\
\hline Número de linhas & 44 & 49 & 49 & 49 \\
\hline Função Objetivo & 70.580 & Não resolve & 77.400 & 65.540 \\
\hline Tempo LINGO (segundos) & 1 & --------- & ---------- & 74 \\
\hline Tempo total (segundos) & 1 & ---------- & 117 & 958 \\
\hline Número de tripulações & 16 & ---------- & 17 & 16 \\
\hline Número de duplas pegadas & 3 & ---------- & 2 & 2 \\
\hline Número de trocas de veículo & 3 & ---------- & 9 & 10 \\
\hline Número de trocas de linha & 0 & ---------- & 7 & 9 \\
\hline Horas extras & 01:10 & ---------- & 00:08 & $00: 54$ \\
\hline Horas ociosas & $27: 57$ & ---------- & $32: 05$ & $26: 11$ \\
\hline
\end{tabular}

Tabela 5: Resultados obtidos usando tempo mínimo de trabalho igual a 4 h00

\begin{tabular}{lccrr}
\hline & Janela de Troca & Agrupamento de Tarefas & Chvátal & Seleção de Colunas \\
\hline Número de colunas & 210 & 460.773 & 460.773 & 46.077 \\
Número de linhas & 44 & 54 & 54 & 54 \\
Função Objetivo & 70.580 & Não resolve & 77.400 & 61.940 \\
Tempo LINGO (segundos) & 1 & -------- & 166 \\
Tempo total (segundos) & 1 & -------- & 117 & 3.122 \\
Número de tripulações & 16 & ------- & 16 \\
Número de duplas pegadas & 3 & -------- & 3 \\
Número de trocas de veículo & 3 & --------- & 2 & 13 \\
Número de trocas de linha & 0 & -------- & 9 & 17 \\
Horas extras & $01: 10$ & ------- & $00: 08$ & $00: 14$ \\
Horas ociosas & $27: 57$ & -------- & $32: 05$ & $25: 31$ \\
\hline
\end{tabular}

Tabela 6: Resultados obtidos usando tempo mínimo de trabalho igual a 3h30

\begin{tabular}{lccrr}
\hline & Janela de Troca & Agrupamento de Tarefas & Chvátal & Seleção de Colunas \\
\hline Número de colunas & 210 & 787.154 & 787.154 & 78.715 \\
Número de linhas & 44 & 58 & 58 & 58 \\
Função Objetivo & 70.580 & Não resolve & 77.400 & 48.910 \\
Tempo LINGO (segundos) & 01 & --------- & 776 \\
Tempo total (segundos) & 01 & -------- & 117 & 8.671 \\
Número de tripulações & 16 & ------- & 17 \\
Número de duplas pegadas & 03 & -------- & 03 \\
Número de trocas de veículo & 03 & -------- & 02 & 14 \\
Número de trocas de linha & 00 & --------- & 09 & 12 \\
Horas extras & $01: 10$ & -------- & $00: 08$ & $00: 47$ \\
Horas ociosas & $27: 57$ & -------- & $32: 05$ & $19: 24$ \\
\hline
\end{tabular}


rado com as demais soluções obtidas).

\section{CONCLUSÕES E CONTINUIDADE DA PESQUISA}

As metodologias propostas mostraram-se capazes de encontrar soluções de qualidade para o problema de programação da tripulação. A janela de troca possui a vantagem de resolver em poucos segundos os problemas propostos. Isto ocorre devido à capacidade deste método de minimizar o número de colunas geradas. Esta é a única metodologia deste trabalho que possui a capacidade de resolver problemas de maiores dimensões em razoável tempo de processamento.

O agrupamento de tarefas mostrou-se eficiente quando usado em conjunto com a seleção de colunas, pois desta forma conseguiu-se obter os melhores resultados para o problema proposto, ou seja, menor número de tripulações e menor quantidade de horas extras e de horas ociosas. A limitação do método se refere ao tamanho do problema que pode ser resolvido.

A heurística de Chvátal mostrou-se capaz de gerar soluções iniciais dispensando tanto o emprego de pacotes de otimização linear quanto o uso de computadores mais potentes.

A continuidade da pesquisa se dará abordando o problema proposto com métodos não exatos, denominados métodos heurísticos, mais precisamente as metaheurísticas, que se destacam por serem métodos inteligentes capazes de escapar de ótimos locais e resolver problemas de grandes dimensões.

Com esta estratégia, espera-se poder comparar as soluções obtidas pelas metaheurísticas e pelos métodos exatos de forma a determinar qual a melhor abordagem para resolver o problema de programação da tripulação de ônibus urbano.

\section{AGRADECIMENTOS}

Os autores agradecem à UFOP pelo apoio dado ao desenvolvimento deste trabalho, bem como a Borland Latin America pela cessão de uma licença de uso do software C++ Builder 6.0.

\section{REFERÊNCIAS BIBLIOGRÁFICAS}

Barnhart, C.; E. L. Johnson; G. L. Nemhauser; M. P. Savelsbergh e P. H. Vance (1998) Branch-and-price: column generation for solving huge integer programs. Operations Research, v.46, p.316-329.

Chvátal, V. (1979) A greedy heuristic for the set covering problem. Mathematics of Operations Research, v. 4, p. 233-235.

Desrochers, M. e F. Soumis (1989) A Column Generation approach to the urban transit crew scheduling problem. Transportation Science, v. 23, p. 1-13.

Desrochers, M.; J. Gilbert; M. Sauve e F. Soumis (1992) CREW-OPT: Subproblem modeling in a column generation approach to urban crew scheduling. In: Computer-Aided Transit Scheduling, Desrochers, M. \& Rousseau, J. M. (eds.), Spring, Berlin, p. 395-406.

Fores, S.; L. Proll e A. Wren (1999) An improved ILP system for driver scheduling. In: Computer-Aided Transit Scheduling, Wilson, N. H. M. (ed.), Springer, Berlin, p. 43-61.
Fores, S. (1996) Column generation approaches to bus driver scheduling. Ph.D. Thesis, University of Leeds.

Fores, S.; L. Proll e A. Wren (2002) TRACS II: a hybrid ip/heuristic driver scheduling system for public transport. Journal of the Operational Research Society 53, 1093-1100.

Freling, R.; D. Huisman e A. Wagelmans (2001) Applying an integrated approach to vehicle and crew Scheduling in practice. In: Voss S. e J. Daduna (eds.), Computer-Aided Scheduling of Public Transport, Lecture Notes in Economics and Mathematical Systems, v. 505, p. 73-90. Springer.

Friberg, C. e K. Haase (1999) An exact branch and cut algorithm for the vehicle and crew scheduling problem. In: Computer-Aided Transit Scheduling, Wilson, N. H. M. (ed.), Springer, Berlin, p. 63-80.

Haase, K.; G. Desaulniers e J. Desrosriers (2001) Simultaneous vehicle and crew scheduling in urban mass transit systems. Transportation Science 35(3), 286-303.

Pedrosa, D. and M. Constantino. (2001). Days-off scheduling in public transport companies. In S. Voss and J. Daduna (eds.), ComputerAided Scheduling of Public Transport, Lecture Notes in Economics and Mathematical Systems, v. 505, p. 215-232. Springer.

Rousseau, J. M. e J. Y. Blais (1985) HASTUS: an interactive system for buses and crew scheduling. In: Computer scheduling of public transport 2, J. M. Rousseau (ed.), 45-60, North-Holland.

Smith, B. M. e A. Wren (1988) A bus crew scheduling system using a set covering formulation. Transportation Research, v. 22A, p.97108.

Valouxis, C. e E. Housos (2002) Combined bus and driver scheduling. Computers and Operations Research 29(3), 243-259.

Wren, A.; R. S. K. Kwan e M. E. Parker (1994) Scheduling of rail driver duties. In: Computers in Railways IV, vol. 2, Murty, T. K. S.; B. Mellitt; C. A. Brebbia; G. Sciutto e S. Sone (eds.), Southampton, Boston.

Yunes, T.; A. Moura e C. de Souza. (1999). Solving Large Scale Crew Scheduling Problems with Constraint Programming and Integer Programming. Technical Report, University of Campinas. 